David VRYDAGHS, «La réception des produits culturels », @nalyses, hiver 2007

\title{
David VRYDAGHS
}

\section{La réception des produits culturels}

Comme l'indique son sous-titre, Actualités des recherches en sociologie de la réception et des publics, cet ouvrage collectif, publié sous la direction d'Isabelle Charpentier à la suite d'un colloque international et pluridisciplinaire organisé en novembre 2003 par le Centre de recherches en sciences politiques de l'Université de Versailles/Saint-Quentin-enYvelines, a pour ambition première de fournir un état des lieux, aussi complet que possible, des études de réception engageant des publics « réels » (par opposition aux esthétiques de la réception qui recourent souvent à un artefact, tel le lecteur modèle d'Umberto Eco). Il réunit à cet effet des chercheurs issus de plusieurs traditions disciplinaires de la sociologie européenne. Sont notamment représentées les Cultural Studies britanniques, mais aussi la sociologie des médias ou encore la sociologie française de la culture et de la littérature. Les orientations méthodologiques retenues (enquêtes statistiques, enquêtes qualitatives, focus groups, analyses de corpus, etc.), les produits culturels choisis (émissions radiophoniques ou télévisuelles, textes littéraires, magazines, articles de presse, etc.) et les publics rencontrés (fans, lecteurs réels, lecteurs supposés, lecteurs professionnels, etc.) accentuent encore la diversité des points de vue portés sur l'objet "réception », assurant ainsi à la plupart des courants une représentation dans ce concert d'études.

Autrement dit, rien ne vient nécessiter ce rassemblement, sinon l'envie de produire une photographie des différentes tendances et outils de recherche disponibles dans le domaine des études de réception, en plein essor depuis quelques années déjà. L'heure n'est pas en effet au manifeste théorico-méthodologique, mais à la cohabitation des regards, diversement informés et instrumentalisés, sur ce qu'Isabelle Charpentier nomme joliment "la "terra incognita" en aval des procès de communication » (p. 6). 
En cela, le livre est plutôt réussi, même si on peut regretter l'absence quasi complète de débat dans cette juxtaposition encyclopédique et œcuménique de travaux. On notera, à titre d'exception, l'échange passionnant entre deux éminents représentants des Cultural Studies, David Morley et Martin Barker, le premier défendant l'intérêt heuristique du modèle de l'encodage/décodage ${ }^{1}$ quand le second soutient au contraire que celui-ci est dépassé. On passe en effet d'une interprétation du poids de l'appartenance de classe dans l'appréciation et l'appropriation d'un feuilleton radiophonique par quelques-uns de ses fans réunis pour l'occasion en focus groups (Lyn Thomas, à propos de l'émission The Archers de la BBC) à l'analyse des différents mécanismes et structures, internationaux comme nationaux, présidant à la réception française d'une littérature étrangère (Gisèle Sapiro, à propos des lettres hébraïques traduites et critiquées en France depuis la Seconde Guerre mondiale).

En revanche, on demeure dubitatif dès lors que l'ambition de départ se mue en volonté de proposer une «cumulativité raisonnée des principaux apports et résultats» (p. 6). La diversité des approches proposées comme la variété des produits culturels et des publics étudiés nuisent en réalité à cet objectif, la question de la cumulativité ne pouvant se poser et se penser qu'au sein d'une même discipline, et non dans un foisonnement interdisciplinaire incontrôlé.

À côté de cette double ambition, partiellement réalisée, ce recueil poursuit un troisième objectif: celui de renforcer, par une série d'études de cas, une tendance actuelle des études de réception consistant à considérer les « récepteurs » comme des acteurs de la réception, et non plus comme des réceptacles privés de marge de manœuvre dès lors qu'ils ont été exposés à des produits façonnés pour eux. Dans le

\footnotetext{
${ }^{1}$ Proposé par Stuart Hall en 1973, ce modèle, adapté notamment à l'étude des réceptions des émissions télévisuelles, signale que toute réception se prépare dès l'encodage du message en fonction d'un public-cible supposé et se poursuit par des décodages multiples et parfois contradictoires. Il est particulièrement intéressant en ceci qu'il met en évidence la marge de manœuvre dont bénéficient les lecteurs ou les spectateurs d'un produit culturel dans leur appréhension de cet objet.
} 
même ordre d'idée, il s'agit de mettre l'accent, à la suite des Cultural Studies, sur les usages concrets des produits culturels, éminemment variables selon les récepteurs et les contextes d'appropriation. Une pensée de l'historien du livre Roger Chartier, citée en introduction, résume bien cet enjeu: "Toujours ces pratiques sont créatrices d'usages ou de représentations qui ne sont aucunement réductibles aux volontés des producteurs de discours et de normes [...]. L'acceptation des modèles et des messages proposés s'opère à travers des aménagements, des détournements, parfois des résistances, qui manifestent la singularité de chaque appropriation. » (p. 12)

Là encore, on observe un décalage frappant entre l'ambition de départ et le résultat final. La plupart des contributions s'intéressent peu aux différents publics des produits culturels. Souvent, seuls les critiques spécialisés retiennent l'attention des chercheurs. Une contribution se passe même d'analyser les réactions du public pour se concentrer sur les stratégies mises en place par les auteurs (Rémy Ponton à propos des frères Goncourt, intervention au demeurant intéressante en ceci qu'elle met en évidence combien ces écrivains ont continuellement livré, dans leur Journal, une réflexion «sur les goûts et le niveau intellectuel du public, comme sur le rôle des instances de légitimation», p. 59). Quand le public « réel» et non professionnel d'un produit est approché, il l'est principalement au travers des chiffres de vente (comme c'est le cas dans la contribution de Fabrice Thumerel, à propos des œuvres théâtrales de Valère Novarina). En fait, seule une section sur les quatre que contient l'ouvrage traite réellement de cette question. Intitulée «Du public aux publics », elle rassemble certes des contributions de qualité (on pense surtout à l'analyse par Isabelle Charpentier du courrier des lecteurs et lectrices de Passion simple, d'Annie Ernaux, qui fait ressortir l'importance de l'appartenance sexuelle dans les appréciations changeantes de l'œuvre), mais ne permet guère de se faire une idée précise de la variété réelle des publics d'une même œuvre (en chaque cas, l'échantillon étudié est faiblement représentatif par rapport à l'ensemble des récepteurs). En somme, ce livre présente moins des appropriations à chaque fois singulières d'objets culturels par différents publics que différentes façons scientifiques et critiques d'approcher ces publics. L'objectif annoncé n'est 
malheureusement pas atteint. C'est là le prix à payer quand on choisit la diversité au détriment de la défense et illustration d'une série contrôlée d'hypothèses de travail.

Une dernière volonté présidait à ce rassemblement des tendances actuelles des études de réception: fournir des outils aux sciences politiques dans leur appréhension des attitudes des publics face aux messages politiques qui leur sont proposés. Ces outils apparaissent çà et là dans l'ouvrage, notamment dans la section approchée précédemment, mais on peut encore une fois se demander s'il n'aurait pas mieux valu concentrer le tir autour de cet objectif. Le choix de la disparité, assumé par l'organisatrice pour «mieux éclairer les formes et les contours actuels des études de réception » (p. 7), fait en fin de compte beaucoup d'ombre aux autres propositions, plus originales celles-là, et qui auraient mérité à coup sûr des développements plus nombreux et plus contrôlés.

Référence : Isabelle Charpentier (dir.), Comment sont reçues les cuvves. Actualités des recherches en sociologie de la réception et des publics, Grâne (France), Créaphis, 2006, 285 p. 